\title{
Bridging Audio and Augmented Reality towards a new Generation of Serious Audio-only Games
}

\author{
Emmanouel Rovithis ${ }^{1}$, Andreas Floros ${ }^{1}$, Nikos Moustakas ${ }^{1}$, Konstantinos Vogklis ${ }^{1}$ and Lily Kotsira ${ }^{2}$ \\ ${ }^{1}$ Ionian University, Department of Audio and Visual Arts, Corfu, Greece \\ ${ }^{2}$ Hellenic-American Educational Foundation, Athens, Greece \\ emrovithis@ionio.gr \\ floros@ionio.gr \\ a11mous@ionio.gr \\ voglis@cs.uoi.gr \\ kotsiralilly@yahoo.com \\ DOI: 10.34190/JEL.17.2.07
}

\begin{abstract}
Educational practices are constantly adjusting to technological advances, in order to improve their effectiveness in delivering knowledge and preparing students for the challenges of modern digital society. Electronic games and augmented reality environments are two such media that can shape powerful modes of interactive and immersive experiences. Audio games in particular, i.e. electronic games that utilise data sonification and audio interaction techniques to express all narrative and gameplay content, have been proven to enhance players' skills, such as concentration, memory, fantasy, emotional response, perception, data management and cooperation. However, despite the promising research findings reported in literature, there has been no systematic approach in integrating systems based on audio interaction into formal education. This article suggests that audio games can be designed and implemented for the delivery of targeted curricula through an engaging learning experience, whereas fusing audio game mechanics into new interactive technologies, such as augmented reality environments, will further enhance the students' immersiveness in the learning process. Towards establishing the theoretical framework for the design of educational audio games, as well as educational augmented reality audio games, the authors review existing approaches and argue that the inherent features of both audio interaction and augmented reality systems agree with the official educational goals formulated by the Greek Ministry of Education and the International Baccalaureate Institution. Then, they discuss four original projects in terms of sound and mechanics design to demonstrate that audio games can address a wide range of subjects, adopt cross-curricular strategies, facilitate complex modes of interaction, and realise creative, collaborative and inclusive learning activities.
\end{abstract}

Keywords: audio games, serious games, augmented reality, augmented reality audio, audio interaction, music education

\section{Introduction}

Digital applications are becoming increasingly ubiquitous, thus expanding the skills required of modern learners to include information literacy. People who are not trained to cope with this new reality will not have the same opportunities for social integration. For example, employment in the most innovative sectors of the European economy is currently marked by a technological skills gap (European Commission, 2014). Education needs to respond to the imperatives shaped by digital revolution. Yet new technologies exhibit features that challenge traditional schooling: they are customisable and specialised, rely on diverse, interconnected and widely spread sources, and require hands-on action besides theoretical knowledge acquisition (Collins and Halverson, 2018). Learners have also changed: they are multi-tasked and playful, accustomed to new media since childhood, and processing information faster than previous generations (Prensky, 2005). Therefore, new technology-friendly ways must be found to motivate towards learning and provide with joyful and creative schooling environments that safeguard equal opportunities for all.

The utilisation of technology for educational purposes is widely supported in literature. A detailed overview of research on the potential benefits of such strategies is provided by Fu (2013): accessing information efficiently and effectively, overcoming temporal and spatial restrictions, developing critical thinking skills, realising creative and collaborative environments, enhancing student-centered and self-directed practices, and fostering modern educational theories. However, it is also observed that, in contrast to other fields where impact has been more extensive, in education technology-oriented approaches are just beginning to have a presence, mostly in specialized courses, due to factors including the lack in infrastructure, funding, training, and motivation. (Oliver, 2002; Collins and Halverson, 2018) 
This article aims to contribute in addressing this issue by answering the following question: do Audio Games (AG) constitute a suitable medium to be integrated in formal education as systems based on audio interaction towards the efficient delivery of curricula related, but not restricted, to musical subjects? Furthermore, could the merging of AG with a state-of-the-art technology, such as Augmented Reality (AR) into hybrid Augmented Reality Audio Games (ARAG) enhance the interactivity and immersiveness of educational environments? In the scope of their investigation, the authors draw on current literature to establish the theoretical framework for the design of educational AG and ARAG. They argue that it is their inherent characteristics in terms of information and gameplay representation that can shape such audio-interactive media into efficient and motivating educational tools for a wide range of subjects. Then they present a multitude of research findings regarding the positive impact of audio interaction on the learning process and compare them to the learning objectives of official educational institutions concluding that they match. Finally, they present some of their recent and ongoing projects, in order to demonstrate the potential of different implementation approaches towards the creation and enhancement of educational scenarios.

Thus, the article is structured as follows: In Section 2 all involved technological agents, namely electronic games, audio games, augmented reality systems, and augmented reality audio are discussed focusing on the learning merits realised by audio interaction. In Section 3 these merits are compared to the official music and arts educational goals formulated by the Greek Ministry of Education and the International Baccalaureate. Section 4 discusses the implementation of AG systems in various educational scopes, including an ARAG realisation for the promotion of cultural heritage. Finally, Section 4 provides with conclusions and considerations.

\section{Educational aspects of audio interactive systems}

\subsection{Electronic games}

Electronic games, in general, constitute a popular and important part of modern digital culture (Kafai, 2006). They possess motivational power by employing challenging modes of interaction and immersion. Their use in education has exhibited positive results in addressing specific skills, problems, and curricula. According to Griffiths (2002) early research has consistently shown that the augmentation of the learning process with educational computer games raises players' self-esteem, improves their hand-eye co-ordination and reduces their reaction time. Another review of multiple studies over a period of 28 years (Randel et al., 1992) reports the effectiveness of educational computer games compared to conventional classroom instruction in improving students' ability to solve mathematics, physics and biology problems, while exciting their interest and enhancing the absorption and retention of knowledge. More recent technological advances in audiovisual design, hardware performance and networking have expanded the capabilities of educational electronic games in terms of interactivity, feedback, diversity, novelty, and immersiveness. Players/learners are now in the centre of the learning process ; they are required to actively explore their environment, be inquisitive and inventive, think critically and make analytical decisions, as well as collaborate with others, in order to successfully cope with the requested challenges, a process which enhances their perceptual receptiveness, memory and self-esteem (Stapleton, 2004; Susi, Johannesson and Backlund, 2007). They are presented with immersive and realistic scenarios for problem-solving, capable of adjusting to their special learning needs and styles (Sancho et al., 2009). Thus, electronic games have become valid platforms for the application of modern learning theories, including Problem Based Learning, Constructivism, Experiential Learning and the Flow Theory (Kiili, 2005).

Regarding audio interaction within a digital environment, research findings are equally promising. Interfaces based on auditory feedback exhibit great potential for helping users become more proficient at the fine manipulation of complex tools (Franinovic and Serafin, 2013). Perceiving and processing multilayered sonic information develops the ability to manage complex data-patterns. Bishop, Amankwatia and Cates (2008) argue that the utilisation of non-speech sound in learning environments leads to a deeper understanding of the material under study. They provide a multitude of researches supporting that sonic stimuli can reduce competing distractions and retain learners' attention. Focusing on music education, it has been suggested that interactive digital environments can introduce learners to low and high-level aspects of music creation regardless of any pre-existing experience (Wishart, 1992; Seddon, 2007; Berndt, 2011). Nevertheless, the inclusion of either serious games, i.e. games specifically designed for educational purposes, or gamification practices, i.e. the use of game design elements in non-game contexts, is still an emerging trend (Dicheva, et al., 
2015). Further investigation is needed to gather more evidence, a direction, to which the systematic design and implementation of educational AG can contribute.

\subsection{Audio games}

As a sub-genre of electronic games, AG have inherited many of the aforementioned educational properties of game interaction, yet it is audio interaction that lies at the core of their mechanics. AG rely mainly or exclusively on sound to express the game's space, plot and action. Players need to concentrate on their sense of hearing, in order to understand and execute the necessary actions. The game's feedback to those actions is also communicated through sound. In general, the AG sonic content includes speech and non-speech stimuli. The latter can be distinguished in auditory icons, which incorporate a clear, realistic reference to the objects or processes they describe (Gaver, 1986), and earcons, i.e. sounds with symbolic content that the user must decode, in order to perceive the intended message (Blattner, Sumikawa and Greenberg, 1989; Mc Gookin and Brooster, 2011). Additional sonification techniques include parameter mapping (Grond and Berger, 2011), while sound spatialisation is frequently employed for expanding the allowed acoustic information capacity (Röber and Masuch, 2004). All these elements aim at immersing players in an audio-based, sometimes even completely eye-free environment. Initially AG addressed the visually impaired community, yet recently, a trend to make the genre known to the broader public has emerged, mostly accounted for by the development of mobile technologies, which facilitate action to take place independently from the screen.

Audio interaction employed by AG has also been found to have a positive impact on the user, since sound is very efficient in causing emotional responses. Parker and Heerema (2008) argue that feelings and memories are triggered by aural stimuli in ways not possible by visual observation, yet AG need to designed in a less visual way. Other researches focus on AG educational and therapeutic aspects: the ability to enhance memory, concentration, fantasy, and skills acquisition through a less dictatorial process compared to conventional visual practices (Targett and Fernström, 2003; Liljedahl \& Papworth, 2008). The absence of visual stimuli results in increased physical freedom as well. Aural action can take place in a $360^{\circ}$ field around the player, thus enhancing the immersiveness of the experience (Röber and Masuch, 2005). This immersiveness can be further enhanced through new interactive technologies, such as AR.

\subsection{Augmented Reality}

AR enriches the real world by computer-generated content, such as images, videos, audio and textual information, tied to specific locations and objects. A widely accepted definition of an AR system includes the following characteristics: 1) combining real and virtual objects in a real environment; 2) running interactively and in real time; and 3) aligning real and virtual objects with each other in three dimensions (Azuma, 1997; Azuma et al., 2001). In contrast to virtual reality, which attempts to completely seclude users from their surroundings, AR aims to seamlessly blend into the naturally perceived world and enhance users' perception of the physical environment by allowing for interaction with the superimposed virtual elements through movement in space and gestural activity. Due to their potential for multisensory interactive experiences AR systems have been applied in a wide range of fields including advertising, medicine, navigation, touring, industrial manufacturing, military, business, entertainment, and education (Krevelen and van Poelman, 2010).

Regarding the latter, the notion that AR technology can be utilized towards the development of an innovative medium for ubiquitous learning is highly supported in literature. At the start of the current decade, the Horizon Report, published by the New Media Consortium's (NMC's) Emerging Technology Initiative seeking to pinpoint the emerging technologies to have a substantial impact on education and other sectors predicted that AR will be widely adopted, with AR games in particular becoming powerful tools in the hands of educators (Johnson, et al., 2010). Yuen, Yaoyuneyong and Johnson (2011) provide numerous studies demonstrating the positive impact of AR applications within a variety of fields related to education ; AR promotes students' motivation, collaboration, imagination, and determination, and can adjust to various learning styles and situations not feasible through first-hand experience. The authors also identify five approaches in AR education: augmented books, augmented gaming, discovery-based learning, objects modeling, and in-situ skills training. Regarding AR books, Billinghurst, Kato and Poupyrev (2001) have suggested that young children can read books in more interactive and realistic ways when seen through a handheld AR device. Regarding educational AR games, a research utilizing an AR role-playing game on the subject of History requiring players to examine the actual historical sites with a GPS-enabled handheld device concluded that AR creates the conditions for exploration, collaboration, and reflection towards solving problems (Schrier, 2005). Similarly, Lee (2012) suggests that it can be used for raising awareness about cultural heritage sites, and provides further 
examples of AR applications teaching Astronomy, Chemistry, Biology, Mathematics, Geometry, and Physics. Other studies have demonstrated that compared with the traditional educational system AR can boost the performance of learners (Jadeja, Mehta and Sharma, 2016), increase motivation especially among the less academically successful students (Freitas and Campos, 2008), and enhance the comprehension of complicated curricula in higher education (Liarokapis, et al., 2004).

Despite these promising findings, the integration of AR systems into education is still quite challenging due to issues such as the cost of development and maintenance, inadequate strategies for merging with traditional learning methods, and suspicion against new technologies (Lee, 2012). But recent advances in the mobile industry are turning the tide. The current generation of smartphones has the necessary computing power, as well as equipment, such as GPS, compass, accelerometer and other sensors, to facilitate the development of portable AR without the need for bulky equipment. Starting with navigational assistance, a variety of locationbased gaming, social networking and healthcare AR applications is now widely available on mobile platforms. The commercial success of the AR exploration game "Pokemon Go!" is indicative of that growing trend (Ratner, 2016).

\subsection{Augmented Reality Audio}

AG can greatly benefit from merging with AR technologies realized on mobile platforms. Utilizing sound as the carrier of augmentation results in the concept of Augmented Reality Audio (ARA), in which users perceive the surrounding environment in its "pseudoacoustic" form, i.e. the real acoustic environment is captured through microphones, mixed with virtual sound events and returned to the user via headphones. AG practices can shape new ways to augment the environment with narrative content, while AR interaction can enrich AG mechanics with elements such as movement and gestures. Although scarce, existing research on ARA supports its educational prospect, focusing on individual aspects including navigation, immersion, and interaction. Several experiments have shown the successful localization of spatialised sounds in an augmented context (Sodnik, et al., 2006) even for users without any musical training (Holland, Morse and Gedenryd, 2002). Other tests have reported increased scores in users' enjoyment and immersion levels and an increase in playful response and sense of discovery, when exploring ARA environments (Reid, et al., 2005; Vazquez-Alvarez, Oakley and Brewster, 2012); in some cases listeners were so immersed that they could not easily determine the real from the virtual sounds (Härmä, et al., 2004).

ARA systems have been applied in a variety of fields, some of which are indirectly related to education. One such example are ARA museum tours, in which the superimposed audio clips are triggered according to the visitors position and behavior, thus facilitating non-linear exploration and the delivery of tailored information (Bederson, 1995; Hatala, et al., 2004). Another approach is raising people's awareness about a site's historical or cultural character. In an ARA installation at Queens Square in Bristol, England visitors could experience the riots that occurred there in 1831 (Reid, et al., 2005). Other ARA sites include a park (Vazquez-Alvarez, Oakley and Brewster, 2012) and a zoo (Stahl, 2007) that were augmented with audio information placed at specific points of interest. Larger real-world spaces, such as cities, can also be augmented by letting individuals associate audio clips with locations (Rozier, 2000) or inform visually impaired people about useful sites in the vicinity, such as restaurants and shops (Blum, Bouchard and Cooperstock, 2011). Further approaches in applying ARA systems include GPS-based navigation through non-speech spatial audio (Holland, Morse and Gedenryd, 2002), augmentation of an office environment by audio notifications on the status of various activities (Mynatt, et al., 1997), mobile security applications providing spatialised audible alerts as cues for navigation to the location of possible threats (Sundareswaran, et al., 2003), and healthcare applications that use the superimposed audio to motivate users towards exercise (Orin, 2014). Another category consists of ARA games, in which players explore their surroundings to interact with virtually placed sounds controlled by the computer or other players. (Ekman, 2007; Lyons, Gandy and Starner, 2000; Moustakas, Floros and Grigoriou, 2011) Last, a throrough survey of ARA art projects is provided by Mariette (2007).

In the majority of the ARA projects reported in literature the main interaction mechanism is physical movement, since the behavior of the virtual sounds depends only on the user's spatial position. Other than that, the user's participation is limited to passive listening. Exceptions to that rule include the following actions in the proximity of a virtual sound: pushing a button (Moustakas, Floros and Kanellopoulos, 2009), turning the device to specific directions (Hatala, 2004), and tapping the device's surface (Ekman, 2007). A systematic implementation of such modes of interaction can lead to the design of more complex scenarios and challenging game experiences. 


\section{Correlating AG with official educational goals}

The previous section investigated the merits of audio interaction in different fields, including electronic games, audio games, augmented reality and the recently emerged augmented reality audio. But in order to systematically apply AG and ARAG in formal education and provide teachers with appropriate tools for enhancing the learning process, the specific needs of modern learners must be taken into consideration. This section draws on learning objectives of official educational institutions and compares them to the impact of audio interaction on the user. Two sources are investigated: the Program of Music Studies formulated by the Greek Ministry of Education (GME), which reflects the current standards in Greece, and the Primary Years Program on learning Arts formulated by the International Baccalaureate Institution (IB), which expresses a globally-oriented approach to the needs of today's citizens of the world (Pedagogical Institute, 2004; International Baccalaureate, 2005). The findings reported in this section were first presented in (Rovithis, Floros and Kotsira, 2018). To that, a connection to ARAG systems has been added. Research focused mainly on music and arts education due to the fact that these subjects lie closer to the nature of sound, yet other directions, such as cultural awareness, are explored as well.

\subsection{Goals of music education by the Greek Ministry of Education}

The official guide of the GME is organized in three sections that define the goals of music education on crosscurricular, primary and secondary level respectively. Each section provides detailed information including sublevel, cognitive content, goals in terms of knowledge, dexterities, stance and values, elements from the respective curriculum, and suggested activities. Structured spirally it advances from simple to complicated concepts, aiming to adapt to the students' kinetic, perceptive, expressive and communicative skills. All sections were examined to extract the respective educational goals, many of which were found to match the research findings on AG interaction. These are:

- To acquire a positive stance and love towards music

- To develop their acoustic ability

- To observe and discuss about how sounds are produced

- To identify sounds of the natural and urban environment

- To identify and organize simple sonic properties, including amplitude, pitch, timbre, duration, melody, rhythm and structure

- To combine sounds into simple compositions

- To perform simpe rhythmic and melodic progressions

- To perform on rhythmic and melodic instruments

- To concentrate on and internalize sounds

- To memorize patterns to develop their acoustic memory

- To explore, select and organize acoustic sources

- To experiment and improvise creatively

- To design sounds to implement them in complex structures

- To elaborate music composition techniques

- To correlate music and sound with other arts and cognitive subjects

- To cooperate and communicate with responsbility and discipline

- To develop their self-esteem

\subsection{Goals of music education by the International Baccalaureate}

IB is an educational program applied to more than one million students in almost 5000 schools in over 150 countries around the world. Based on the principle that people need an interdisciplinary and international education, IB curriculum aims to foster students' global consciousness and develop their critical thinking, multilinguality and collaborative skills. This article examines the IB Primary Years Program focusing on Arts education. According to IB, arts constitute a powerful mode of communication towards building one's inward and outward perception. Again, it was found that the merits of AG interaction agree with IB regarding the following learning objectives:

- To develop their natural curiosity and become stimulated for challenges

- To understand that arts communicate feelings, ideas and experiences

- To consider works of art from different perspectives 
- To explore new roles, ideas, strategies and artistic presentations

- To critically interpret works of art

- To articulate their thought through a variety of new media and technologies

- To engage their imagination for creative exploration

- To develop their conceptual understanding

- To develop their listening skills

- To describe differences in music

- To synchronize with rhythm

- To classify and analyze sounds

- To play musical instruments

- To create simple compositions

- To participate in collaborative live performances

Table 1 summarizes the findings:

Table 1: Greek Ministry and IB educational goals compared to AG interaction features

\begin{tabular}{|c|c|c|}
\hline $\begin{array}{l}\text { Educational Goals - } \\
\text { Greek Ministry of Education }\end{array}$ & $\begin{array}{l}\text { Educational Goals - } \\
\text { International Baccalaureate }\end{array}$ & $\begin{array}{l}\text { Features of } \\
\text { AG Interaction }\end{array}$ \\
\hline Positive stance towards music & $\begin{array}{l}\text { Stimulation for challenges, } \\
\text { Development of curiosity }\end{array}$ & $\begin{array}{l}\text { Exciting players' interest, } \\
\text { Motivating towards knowledge, } \\
\text { Applying modern learning theories }\end{array}$ \\
\hline Development of self-esteem & & $\begin{array}{l}\text { Raising players' self-esteem, } \\
\text { Reducing negativity }\end{array}$ \\
\hline $\begin{array}{l}\text { Experimentation, } \\
\text { Creative improvisation, } \\
\text { Combination of sounds into simple } \\
\text { compositions, } \\
\text { Elaboration of techniques }\end{array}$ & $\begin{array}{l}\text { Creation of simple compositions, } \\
\text { Exploration of artistic ideas and strategies }\end{array}$ & $\begin{array}{l}\text { Guiding players to experiment and realize } \\
\text { their musical ideas, } \\
\text { Creating platform for music composition } \\
\text { and improvisation }\end{array}$ \\
\hline $\begin{array}{l}\text { Development of acoustic ability, } \\
\text { Identification of sounds, } \\
\text { Identification of sonic properties, } \\
\text { Observation of sound production }\end{array}$ & $\begin{array}{l}\text { Development of listening skills, } \\
\text { Ability to discern musical differences }\end{array}$ & $\begin{array}{l}\text { Introducing players to musical concepts } \\
\text { without prerequisites, } \\
\text { Guiding players to identify sonic } \\
\text { properties, } \\
\text { Employing mechanics that target specific } \\
\text { musical skills }\end{array}$ \\
\hline $\begin{array}{l}\text { Concentration, } \\
\text { Internalization of sounds }\end{array}$ & & $\begin{array}{l}\text { Focusing on the sense of hearing, } \\
\text { Excluding distractions, } \\
\text { Gaining players' attention and retaining it } \\
\text { over time, } \\
\text { Increasing concentration, } \\
\text { Enhancing immersion }\end{array}$ \\
\hline $\begin{array}{l}\text { Cooperation, } \\
\text { Communication }\end{array}$ & $\begin{array}{l}\text { Participation in collaborative } \\
\text { performances }\end{array}$ & $\begin{array}{l}\text { Promoting collaboration among team } \\
\text { members }\end{array}$ \\
\hline $\begin{array}{l}\text { Correlation of music and sound with } \\
\text { other arts and subjects }\end{array}$ & $\begin{array}{l}\text { Consideration of art from different } \\
\text { perspectives }\end{array}$ & Sonification of non-musical data \\
\hline $\begin{array}{l}\text { Sound design into complex structures, } \\
\text { Organization of acoustic sources }\end{array}$ & Classification and analysis of sounds & $\begin{array}{l}\text { Facilitating the exploration of compex } \\
\text { patterns, } \\
\text { Enhancing analytical thought, } \\
\text { Managing complex sets of data }\end{array}$ \\
\hline $\begin{array}{l}\text { Pattern memorization, } \\
\text { Development of acoustic memory }\end{array}$ & & Enhancing memory \\
\hline \multirow[t]{4}{*}{$\begin{array}{l}\text { Performance of rhythmic and melodic } \\
\text { progressions on respective musical } \\
\text { instruments }\end{array}$} & $\begin{array}{l}\text { Playing musical instruments, } \\
\text { Synchronization with rhythm }\end{array}$ & $\begin{array}{l}\text { Enhancing dexterity at fine movements } \\
\text { and manipulation of complex tools } \\
\text { Improving hand-eye coordination }\end{array}$ \\
\hline & $\begin{array}{l}\text { Engagement of imagination for creative } \\
\text { exploration, } \\
\text { Development of conceptual } \\
\text { understanding, } \\
\text { Critical interpretation of artistic works }\end{array}$ & $\begin{array}{l}\text { Allowing freedom for interpretation, } \\
\text { Releasing fantasy }\end{array}$ \\
\hline & $\begin{array}{l}\text { Articulation of thought through new media } \\
\text { and technologies }\end{array}$ & $\begin{array}{l}\text { Employing a familiar technological medium } \\
\text { of modern culture }\end{array}$ \\
\hline & $\begin{array}{l}\text { Understanding that arts communicate } \\
\text { feelings, ideas and experiences }\end{array}$ & Carrying emotional content \\
\hline
\end{tabular}




\subsection{Towards educational ARA systems}

As aforementioned, the systematic use of AR systems in education is a quite promising, but still challenging task. ARA systems in particular have found far fewer applications than their visual AR counterparts. The closest example to implementing ARA systems is found in the design of interactive audio augmented tours, either to provide information about the exhibits, or to raise awareness about the historical or cultural importance of a site. Both the GME and the IB Programs of Studies converge towards these ARA directions.

Regarding GME, as soon as in the Introductory Note of the Cross Curricular/Thematic Framework it is pointed out that the role of school ought to be redefined, in order to meet the needs shaped by the modern information and knowledge society. Education is no longer to be governed by traditional field-centered practices that promote a rather passive attitude towards learning, but instead should adopt cross-thematic organization of content and new, inquiry-based and holistic approaches to delivering knowledge. The following educational goals identified in the General Principles section can be addressed through ARA:

- $\quad$ to develop pupils' skills, abilities and interests

- to provide equal opportunities for learning for all pupils

- to reinforce the pupils' cultural and linguistic identity within the framework of a multicultural society

- to develop environmental awareness and foster relevant patterns of behavior

- to prepare pupils to explore new information and communications technologies

Regarding IB the following properties are important for the desired learner's profile:

- to be capable of perceiving and expressing ideas in a variety of modes of communication

- $\quad$ to understand and appreciate own cultures and personal histories

- $\quad$ to be open to the values and traditions of others

It is also stated that new media and technologies are the tools for imaginative conceptualisation and creative expression. They will not only prepare students for the complexity of modern digital world, but also provide with novel means for engaging them with historical, social and cultural perspectives.

Modern educators can build on the ground made fertile by the multitude of research findings presented, and use the aforementioned officially formulated learning objectives as design guidelines for realising immersive educational scenarios through state-of-the-art ARA technologies. These can then be applied cross-curricularly, expanding from music-related to non-music-related subject matters, and introduce students to a novel technological medium that will motivate them towards the acquisition of knowledge through exciting and challenging game experiences. Some practices to take into consideration in the design process of such scenarios would include:

- to immerse learners in exploring multilayered learning environments

- to utilise meta-data for adjusting personalised experiences to the learning needs

- to engage learners to collaborate for the completion of challenging tasks

- to facilitate complex ways of audio interaction through gestural movement

- to employ sonification techniques for delivering targeted curricula through the auditory channel

\section{Audio gamification approaches}

This section discusses education-related projects developed by the authors. They have been carefully chosen to advocate the versatility of $A G$ as potential educational tools by demonstrating that the genre can benefit from recent technological advances and employ different interaction and sound design techniques to address a variety of subjects. Special consideration is given to describe an educational scenario, which fuses $A G$ mechanics into an AR environment, thus introducing the ARAG field. More specifically:

- "Noize Games" consists of two AG puzzles aiming to raise awareness about the issue of environmental noise

- "Kronos" is an Role-playing AG teaching pronciples and practices of electronic music composition

- "AstroSonic" is an AG environment using haptics to convey information on Earth's atmosphere and the solar system 
- "Audio Legends" is an ARAG aiming to promote the cultural heritage of the island of Corfu in Greece

While some audio mechanics are common in these audio gamification approaches, other elements differentiate them significantly from one another, posing specific challenges that need to be dealt with. Such factors include for instance the subject matter, the characteristics of players' interaction including their collaboration and/or competition, the duration of the suggested activities, and the technological framework for their realisation. Essentially, different gameplay modes address different learning objectives. Table 2 provides an overview of these considerations.

Table 2: Differences between audio gamification approaches

\begin{tabular}{|l|l|l|l|l|}
\hline Noize Games & Subject Matter & Genre & Technology & Activity Specifications \\
\hline Kronos & Puzzle & $\begin{array}{l}\text { mouse \& } \\
\text { keyboard }\end{array}$ & $\begin{array}{l}\text { Game through speakers in the classroom } \\
\text { Learners can collaborate in groups to solve the riddle } \\
\text { Groups can compete each other in speed }\end{array}$ \\
\hline AstroSonic & Astronomy & RPG & $\begin{array}{l}\text { Action } \\
\text { keyboard }\end{array}$ & $\begin{array}{l}\text { Each learner plays alone using headset } \\
\text { Lab-gaming sessions stretch throughout the semester } \\
\text { Players can exchange data packages }\end{array}$ \\
\hline Audio Legends & Culture & Adventure & $\begin{array}{l}\text { Collaboration of sighted and visually impaired learners } \\
\text { Haptic devices and spatialisation techniques to } \\
\text { thoroughly replace visual stimuli }\end{array}$ \\
\hline
\end{tabular}

\subsection{Noize Games}

"Noize Games" (Rovithis, et al., 2014) was developed within the context of the International Noize Awareness Day. It consists of two AG puzzles. "Match Noize" is a Pelmanism-style game, in which the cards to be flipped contain not pictures, but sounds related to various aspects of noise. Players exercise their acoustic memory, while being introduced to the richness of noise as an acoustic phenomenon. "Escape Noise" is a Point-andClick game, in which players are exposed to a noisy soundscape, whose layers are neutralised at specific coordinates. Trying to locate those spots on the screen to achieve silence, players are guided to realise not only stressful noises from the everyday environment, but also subtle noisy textures that are not easily discernible. Both games are designed in respect to the learning objectives. First, the pupils concentrate on noise itself in a laboratory-style activity, where they can listen to the sounds repeatedly and carefully examine their sonic properties; the goal is to acquire a clear understanding of sources and features of noise. Then, the acquired knowledge is applied on a gaming scenario that combines the sounds into stressful soundscapes, from which players have to escape ; the goal here is to raise awareness about the potentially harmful impact of noise on the acoustic environment. Customisable options, such as the available time and the number of attempts allow the educator to adjust the games' difficulty to the needs of the learning group.

In terms of sonification, earcons were designed to indicate the game's feedback to players' actions, such as "right", "wrong", and "option selection". All other sonic content consists of 36 auditory icons that demonstrate the range of noisy sounds. The criteria that a sound has to meet to be characterised as noise are: a) timbre, i.e. its spectral energy is scattered at different frequencies lacking harmonic structure, or b) distortion, i.e. unwanted and disruptive frequencies to a signal, or c) overload, i.e. many similar sound events accumulating energy at a specific frequency range. The sounds were then organised in three groups -domestic, urban, and natural- according to the environment, in which they occur. In "Match Noise" these environments form the three levels of the game, whereas in "Escape Noise" the sounds are further organised in groups of four to form nine soundscapes. For example, one of the urban soundscapes includes sounds from a large crowd protesting, car horns, engines running and a train passing. 


\subsection{Kronos}

"Kronos" (Rovithis, 2015) adopts the structure of role-playing games: players accomplish various AG tasks that exercise different musical skills to gain respective sound production modules as rewards. They are free to make choices regarding the path they follow and the challenges they complete, which will essentially define the modular structure of their instrument. They are also provided with a dynamic interface to experiment with their instrument-under-construction by inserting audio material, customising settings, and practicing their performance.

The game is intended to address pupils of upper secondary education and students of tertiary education in the scopes of raising their awareness about electronic sound in terms of synthesis and aesthetics, and facilitating their creative expression through electronic media. Players are provided with the option to save the settings and audio material of their avatar and share it with other players. Educators are suggested to run the game in a series of sessions and focus each time on a different part of the curriculum and the respected mechanics. These include identifying sonic properties, such as pitch and timbre, describing the tonal progression of a melody, memorising and repeating rhythmic patterns, navigating through soundscapes in search of hidden sounds, and aiming at sonic objects using ear-hand synchronization. It is recommended that players are given in each session enough time to experiment on their ideas. They can also be guided towards a collaborative performance using the game as their musical instrument.

In terms of sonification, apart from some earcons and auditory icons, parameter mapping was mainly employed. The essence of being introduced to musical concepts, while following the narrative of a game, is that a system of symbolisms between agents of the curriculum and the scenario is established. For example, the game's villain is white noise sonifying Kronos himself: it absorbs all frequencies just as the ancient Greek God was absorbing his own children. Another example would be the choice that players are required to make regarding their role in the game, a choice which radically shapes the nature of their instrument. As Ghosts, creatures of the Underworld, players use noise as raw material for their synthesis engine and focus on respective techniques, such as applying filters on noisy spectra. As Spirits, creatures harnessing the power of the soul, players harness pure tones, a material equally invisible from the real world, and focus on techniques related to oscillators and their modulation. Last, as Humans, creatures driven by the mortality of their flesh, players feed their synthesis engines with recorded instances of the real world and thus focus on the ways of sampling. "Kronos" is built on such a system of symbolisms that maps all narrative elements onto musical material, processes and concepts.

\subsection{AstroSonic}

"AstroSonic" (Rovithis and Floros, 2018) investigates the potential of AG interaction to convey information not related to music or acoustics. More specifically, the game deals with Astonomy, aiming to inform about features of our solar system, such as the size, orbit, and atmosphere of planetary objects, and to raise awareness about the challenges of space exploration, such as asteroid mining and space debris. Players must focus on sonic properties to accomplish the necessary tasks, whereas controllers, such as joystick, mouse, distance and pressure sensors, knobs and faders, facilitate haptic interaction with the game engine. Game mechanics include identifying the properties of sonic objects, such as their spectral characteristics, rhythmic organisation, and stereo position and movement, in order to navigate a spaceship, avoid solar storms, collect ores and debris, and orbit and visit planetary objects. Furthermore, the gameplay was designed in respect of accessibility and collaborative learning. Regarding the former, audio-only interaction realised through a haptic interface makes the game fully accessible for pupils with visual impairment, thus facilitating their inclusion in the learning process. Regarding the latter, a multi-player option is provided, in which pupils play in groups, with each member having to cope with their own tasks in coordination with the rest of the crew.

In terms of sonification, feedback information is conveyed through auditory icons representing mechanical processes from the spaceship's interior and thus enhancing players' immersion in the game environment. All other space elements - space is silent, so realism was not an option here - are sonified through earcons. This distinction between auditory icons and earcons is helpful for the perceptual organisation of the game's sonic content, since realistic sounds refer to players' actions and their results, whereas symbolic sounds deliver the scientific data under examination. The multiple facets of astronomical information are sonified through parameter mapping. For example, the type of a piece of space debris is expressed through the timbre of the respective sonic object, its azimuth position through panning, elevation through a combination of pitch and 
filtering, and proximity through a combination of amplitude and radar-like rhythmic behavior. Familiar conventions among these sonifications allow players' focus to shift from decoding the game's communication language to understanding and executing the necessary tasks.

\subsection{Audio Legends}

"Audio Legends" is a work-in-progress aiming to investigate the implementation of AG mechanics in an AR environment. The game is designed for mobile devices and utilises geolocated information retrieval technology to track user's position in relation to virtual objects on the spatial map. Additionally, it incorporates a novel approach for ARA mixing based on psychoacoustic criteria. (Moustakas, et al., 2019) Positive empirical results will further enhance the -so far theoretical- suggestion by the authors that ARAG systems can be successfully applied for educational purposes. Towards the development of this novel field, "Audio Legends" draws on the findings reported in the previous sections and aims at the education-related task of raising people's awareness about cultural heritage.

The game's scenario is based on the local legends about Saint Spyridon, the protector of the island of Corfu. According to tradition, the saint saved the island from destruction on four different occasions. First, in the mid$16^{\text {th }}$ century he put an end to famine by guiding three Italian ships carrying wheat out of a storm into the harbour. Then, during the $17^{\text {th }}$ century he intervened twice to abolish the deadly plague. Finally, in the early $18^{\text {th }}$ century he stopped the siege of the island by scaring the Turkish fleet away. Key component of the design process was to include as many elements as possible from these folk stories in the game's narrative and mechanics.

In that scope, three game modes were designed to address the saint's labours respectively: 1) St. Spyridon and the Famine, 2) St. Spyridon and the Plague, and 3) St. Spyridon and the Siege. All three modes are composed of two phases. The first phase is similar in all, requiring players to navigate towards a virtual sound source. It is called "Exploration Phase" and is built on standard ARA practices facilitating navigation within the digitally augmented physical space and localisation of superimposed sonic objects by communicating distance and direction to a destination through the processing of amplitude and panning. The second phase is activated, when the user enters the near space surrounding the virtual source. In this "Interaction Phase" the three game modes differentiate themselves considerably from one another aiming to investigate different modes of interaction. More specifically:

- In "St. Spyridon and the Famine" players first locate the sound of the Italian sailors (Exploration Phase) and then guide it with their voice to the entrance of the castle by speaking a certain phrase to the microphone of their device everytime the sound stops following them (Interaction Phase).

- In "St. Spyridon and the Plague" players must first locate the sound of the plague (Exploration Phase), but they can listen to the virtual sound, only if they turn their device to the sound's direction. Then, they must fight the sound off by pointing their device at it and pushing a virtual button (Interaction Phase).

- In "St. Spyridon and the Siege" players first locate the sounds of the Turkish ships (Exploration Phase) that this time are heard simultaneously. To neutralise them, they have to enter each one's proximity and use their device to draw a specific shape in the air (Interaction Phase).

The Exploration Phase seeks to confirm the positive results reported by current literature regarding navigation through audio feedback, whereas the Interaction Phase enters uncharted area aiming to provide new findings about complex interaction mechanisms that can emerge by fusing AG with AR. Both phases will be evaluated in the near future in terms of immersion and educational effectiveness through quantitative and qualitative methods.

\section{Conclusions}

This article has argued for the systematic implementation of educational AG and ARAG towards enhancing the learning process. To establish the theoretical framework of the field the authors conducted investigation on: a) the impact of interaction, in general, and audio interaction, in particular, of digital agents including electronic games, audio games, augmented reality, and augmented reality audio systems, b) the education-related directions, in which audio-interaction systems have been employed, and c) the relevance of the reported findings in literature to the official learning objectives of educational institutions (GME and IB). It has been concluded that the merits of audio interaction within a game environment meet the standards of the officially formulated educational goals to an extent that their integration in formal education is strongly supported. 
Four such implementation approaches have been suggested by the authors demonstrating that AG can be designed to address different subjects, such as acoustics, music, astronomy, and cultural heritage, provide with creative, collaborative, and inclusive learning environments, and benefit from technological advances to realise complex and immersive modes of interaction, such as haptics and voice input.

Investigation revealed some other issues as well. There has been no systematic approach in designing AG in an aural rather than visual way and with a clear educational orientation. Adopting AR systems in schools is subject to infrastructural and social restrictions. ARA systems in particular have been scarcely used for educationrelated purposes and mostly rely on passive interaction rather than on complex interaction mechanisms. The authors suggest that AG be specially designed with audio interaction and learning objectives as the main considerations. The task is not an easy one ; the balance between functionality and aesthetics, and between instruction and dramaturgy has to be found. Challenging game scenarios targeting the learning material in cross-curricular ways should be implemented. Traditional modes of audio interaction, such as hand-ear coordination, rhythmic synchronisation and the identification of sonic properties, must be enriched by stateof-the-art technologies in the scope of enhancing students' motivation and creating immersive learning experiences. This article could serve as framework and inspiration for future developments towards those goals.

\section{Acknowledgements}

This work is funded by the Operational Program Human Resources Development, Education and Life- long learning, Priority Axes 6, 8, 9, Act "Supporting Researchers with emphasis on New Researchers" (grant MIS number: 5007016), which is co-financed by Greece and the European Union (European Social Fund - ESF).

\section{References}

Azuma, R.T., 1997. A Survey of Augmented Reality. A survey of augmented reality. Presence: Teleoperators \& Virtual Environments, 6(4), pp.355-385.

Azuma, R., Baillot, Y., Behringer R., Feiner, S., Julier, S. and Maclntyre, B., 2001. Recent advances in augmented reality, Naval Research Lab, Washington DC.

Bederson, B.B., 1995. Audio augmented reality: a prototype automated tour guide. In: Conference companion on Human factors in computing systems. ACM, pp. 210-211.

Berndt, A., 2011. Diegetic Music: New Interactive Experiences. Game Sound Technology and Player Interaction: Concepts and Developments, 60-76.

Billinghurst, M., Kato, H. and Poupyrev, I., 2001. The magicbook-moving seamlessly between reality and virtuality. IEEE Computer Graphics and applications, 21(3), pp.6-8.

Bishop, M. J., Amankwatia, T. B. and Cates, W. M., 2008. Sound's use in instructional software to enhance learning: A theory-to-practice content analysis. Educational Technology Research and Development, 56(4), 467-486.

Blattner, M. M., Sumikawa, D. A. and Greenberg, R. M., 1989. Earcons and icons: Their structure and common design principles. Human-Computer Interaction, 4(1), 11-44.

Blum, J. R., Bouchard, M. and Cooperstock, J.R., 2011. What's around me? Spatialized audio augmented reality for blind users with a smartphone. In: International Conference on Mobile and Ubiquitous Systems: Computing, Networking, and Services. Springer, Berlin, Heidelberg, pp. 49-62.

Collins, A. and Halverson, R., 2018. Rethinking education in the age of technology: The digital revolution and schooling in America, Teachers College Press.

Dicheva, D., Dichev, C., Agre, G. and Angelova, G., 2015. Gamification in education: A systematic mapping study. Journal of Educational Technology \& Society, 18(3).

Ekman, I., 2007. Sound-based Gaming for Sighted Audiences-Experiences from a Mobile Multiplayer Location Aware Game. In: Audio Mostly 2007. p. 148.

European Commission, 2014. Education Training Youth and Sport. [online] European Union. Available at: <https://europa.eu/european-union/topics/education-training-youth_en> [Accessed 8 February 2019].

Franinović, K. and Serafin, S., 2013. Sonic interaction design, Mit Press.

Freitas, R. and Campos, P., 2008. SMART: a SysteM of Augmented Reality for Teaching 2nd grade students. In: 22nd British $\mathrm{HCl}$ Group Annual Conference on People and Computers: Culture, Creativity, Interaction-Volume 2. BCS Learning \& Development Ltd., pp. 27-30.

Fu, J.S., 2013. ICT in education: A critical literature review and its implications. International Journal of Education \& Development using Information \& Communication Technology, 9(1).

Gaver, W. W., 1986. Auditory icons: Using sound in computer interfaces. Human-Computer Interaction, 2(2), 167-177.

Griffiths, M., 2002. The educational benefits of videogames. Education and Health, 20(3), 47-51.

Grond, F., and Berger, J., 2011. Parameter mapping sonification. The Sonification Handbook, 363-397.

Härmä, A., Jakka, J., Tikander, M., Karjalainen, M., Lokki, T., Hiipakka, J. and Lorho, G., 2004. Augmented reality audio for mobile and wearable appliances. Journal of the Audio Engineering Society, pp.52(6), 618-639. 
Hatala, M., Kalantari, L., Wakkary, R. and Newby, K., 2004. Ontology and rule based retrieval of sound objects in augmented audio reality system for museum visitors. In: ACM Symposium on Applied Computing (SAC'04). pp. 10451050. Available at: http://portal.acm.org/citation.cfm?doid=967900.968114.

Holland, S., Morse, D. R. and Gedenryd, H., 2002. AudioGPS: Spatial Audio Navigation with a Minimal Attention Interface. Personal and Ubiquitous Computing, pp.6(4), 253-259.

International Baccalaureate, 2005. [online] available at: <https://www.ibo.org $>$ [Accessed 8 February 2019)

Jadeja, A., Mehta, R. and Sharma, D., 2016. New era of teaching learning: 3D Marker based augmented reality. International Journal of Information, 6(1/2).

Johnson, L., Levine, A., Smith, R. and Stone, S., 2010. The 2010 Horizon Report. Austin, Texas: The New Media Consortium.

Kafai, Y. B., 2006. Playing and making games for learning instructionist and constructionist perspectives for game studies. Games and Culture, 1(1), 36-40.

Kiili, K., 2005. On educational game design: building blocks of flow experience, Ph. D. Tampere University of Technology.

Krevelen, D.W.F. van and Poelman, R., 2010. A survey of augmented reality technologies, applications and limitations. International journal of virtual reality, 9(2), 1.

Lee, K., 2012. Augmented reality in education and training. TechTrends, 56(2), pp.13-21.

Liarokapis, F., Mourkoussis, N., White, M., Darcy, J., Sifniotis, M., Petridis, P., Basu, A. and Lister, P. F., 2004. Web3D and augmented reality to support engineering education. World Transactions on Engineering and Technology Education, 3(1), pp.11-14.

Liljedahl, M., and Papworth, N., 2008. Beowulf field test paper. In: Audio Mostly, 43.

Lyons, K., Gandy, M. and Starner, T., 2000. Guided by Voices: an Audio Augmented Reality System. Georgia Institute of Technology.

Mariette, N., 2007. From Backpack to Handheld - the Recent Trajectory of Personal Location Aware Spatial Audio. In: 7th digital arts and culture conference. Curtin University of Technology, Perth. pp. 233-240.

McGookin, D. and Brewster, S., (2011) Earcons.

Moustakas, N., Floros, A. and Kanellopoulos, N. G., 2009. Eidola: an Interactive Augmented Reality Audio-Game Prototype. In: Audio Engineering Society Convention 127. Audio Engineering Society.

Moustakas, N., Floros, A. and Grigoriou, N., 2011. Interactive audio realities: An augmented/mixed reality audio game prototype. In: Audio Engineering Society Convention 130. Audio Engineering Society.

Moustakas, N., Floros, A., Rovithis, E. and Vogklis, K., 2019. (in press) Augmented Audio-only Games: A New Generation of Immersive Acoustic Environments through Advanced Mixing. In: 146th Convention of the Audio Engineering Society. AES (Accepted for publication November 2018)

Mynatt, E. D., Back, M., Want, R. and Frederick, R., 1997. Audio Aura: Light-weight audio augmented reality. In: 10th annual ACM symposium on User interface software and technology. ACM, pp. 211-212.

Oliver, R., 2002. The role of ICT in higher education for the 21st century: ICT as a change agent for education,

Orin, A., 2014. Behind the App: The Story of Zombies, Run! Lifehacker. [online] Available at: <https://lifehacker.com/behind-the-app-the-story-of-zombies-run-1632445358> [Accessed 9 September 2018].

Parker, J. R. and Heerema, J., 2008. Audio interaction in computer mediated games. In: International Journal of Computer Games Technology, 2008, 1.

Pedagogical Institute (2004) A Cross-thematic Curriculum Framework. [online] Available at: http://www.pischools.gr/programs/depps/ [Accessed 9 September 2018].

Prensky, M., 2005. Computer games and learning: Digital game-based learning. Handbook of computer game studies, 18, pp.97-122.

Randel, J. M., Morris, B. A., Wetzel, C. D., and Whitehill, B. V., 1992. The effectiveness of games for educational purposes: A review of recent research, Simulation \& Gaming, 23(3), 261-276.

Ratner, P., 2016. What Is Pokémon Go and Why You Should Care. [online] Big Think. Available at: <https://bigthink.com/paul-ratner/what-is-pokemon-go-and-why-you-should-care> [Accessed September 9, 2018].

Reid, J., Geelhoed, E., Hull, R., Cater, K. and Clayton, B., 2005. Parallel Worlds: Immersion in Location-Based Experiences. In: CHI'05 extended abstracts on Human factors in computing systems. ACM, pp. 1733-1736.

Röber, N. and Masuch, M., 2004. Interacting With Sound: An Interaction Paradigm for Virtual Auditory Worlds. ICAD.

Röber, N. and Masuch, M., 2005. Leaving the screen: New perspectives in audio-only gaming. In: 11th Int. Conf. on Auditory Display ICAD. Citeseer.

Rovithis, E., Floros, A., Mniestris, A. and Grigoriou, N., 2014. Audio games as educational tools: Design principles and examples. In: Games, Entertainment, \& Media (GEM). IEEE. pp. 16-23.

Rovithis, E., 2015. Kronos: Electronic Audio Game based on Electronic Music Composition in Educational Applications. Ph. D. Ionian University

Rovithis, E. and Floros, A., 2018. AstroSonic: an educational audio gamification approach. In: DCAC Conference, Interdisciplinary Creativity in Arts and Technology. Ionian University.

Rovithis, E., Floros, A. and Kotsira L., 2018. Educational Audio Gamification: Theory and Practice. In: European Conference on e-Learning (ECEL). ACPI, pp. 497-505.

Rozier, J.M., 2000. Hear\&There: an Augmented Reality System of Linked Audio. Ph. D. Massachusetts Institute of Technology.

Sancho, P., Moreno-Ger, P., Fuentes-Fernández, R. and Fernández-Manjón, B., 2009. Adaptive Role Playing Games: An Immersive Approach for Problem Based Learning. Journal of Educational Technology \& Society, 12(4). 
Schrier, K.L., 2005. Revolutionizing history education: Using augmented reality games to teach histories. Ph. D. Massachusetts Institute of Technology.

Seddon, F. A., 2007. Music e-Leaming Environments: Young People, Composing and the Internet. Music Education with Digital Technology, 107.

Sodnik, J., Tomazic, S., Grasset, R., Duenser, A. and Billinghurst, M., 2006. Spatial sound localization in an augmented reality environment. In: 18th Australia conference on computer-human interaction: design: activities, artefacts and environments. ACM, pp. 111-118.

Stahl, C., 2007. The roaring navigator: a group guide for the zoo with shared auditory landmark display. In: 9th international conference on Human computer interaction with mobile devices and services. ACM, pp. 383-386.

Stapleton, A. J., 2004. Serious games: Serious opportunities. In: Australian Game Developers Conference, Academic Summit, Melbourne.

Sundareswaran, V., Wang, K., Chen, S., Behringer, R., McGee, J., Tam, C. and Zahorik, P., 2003. 3D audio augmented reality: implementation and experiments. In: 2nd IEEE/ACM international symposium on mixed and augmented reality. IEEE Computer Society, p. 296.

Susi, T., Johannesson, M. \& Backlund, P., 2007. Serious games: An overview.

Targett, S. and Fernström, M., 2003. Audio games: Fun for all? All for fun. In: ICAD.

Vazquez-Alvarez, Y., Oakley, I. and Brewster, S.A., 2012. Auditory display design for exploration in mobile audio-augmented reality. Personal and Ubiquitous computing, pp.16(8), 987-999.

Wishart, T., 1992. Music and Technology: problems and possibilities. Companion to Contemporary Musical Thought, 1, 565-582.

Yuen, S.C.-Y., Yaoyuneyong, G. and Johnson, E., 2011. Augmented reality: An overview and five directions for AR in education. Journal of Educational Technology Development and Exchange (JETDE), 4(1), 11. 